\title{
Dipole Polarization Formed on Surface of Polypropylene Electrets
}

\author{
Yasuhiro Matsuda \\ Shizuoka University \\ Department of Materials Science and Chemical Engineering \\ 3-5-1 Johoku, Naka-ku, Hamamatsu, 432-8561, Japan. \\ Yoshihiro Saito \\ Shizuoka University \\ Department of Materials Science and Chemical Engineering \\ 3-5-1 Johoku, Naka-ku, Hamamatsu, 432-8561, Japan. \\ and Shigeru Tasaka \\ Shizuoka University \\ Department of Materials Science and Chemical Engineering \\ 3-5-1 Johoku, Naka-ku, Hamamatsu, 432-8561, Japan.
}

\begin{abstract}
The surface chemical composition and pyroelectric properties in polypropylene (PP) film poled by corona discharge in the air were estimated by Fourier transform infrared spectrophotometry (FTIR), gel permeation chromatography (GPC), differential scanning calorimetry (DSC), thermally stimulated depolarization current (TSDC), and pyroelectricity measurement. The corona discharge oxidized the PP chains at the surface into oligomers bearing $\mathrm{C}=\mathrm{O}, \mathrm{C}-\mathrm{O}$ and $\mathrm{COH}$ groups, and formed hetero charge, and counter homo charge absorbed strongly on the surface. The pyroelectricity and surface potential were undetected after removing the polymer surface with solvent or scratching. TSDC curves indicated that the both homo and hetero charges were stable below the melting point of the oligomers. The stabilization mechanism of surface potential was investigated from the electric behavior and the chemical structure of poled PP films.
\end{abstract}

Index Terms - Electrets, polypropylene films, surface treatment, dipole arrays, corona.

\section{INTRODUCTION}

THE surface polarization of film electrets can induce both same and opposite charge as the previously applied electric field, and the former and the latter are called as "homo charge" and "hetero charge", respectively. While the homo charge is generally assigned to true charge injected from the film surfaces, the hetero charge is identified with the formation or orientation of dipoles or the extraction of opposite excess charge in the whole region of the film. There have been many researches on the electrets with both home and hetero charges [1-4], although little attention was paid to the change of chemical structure inducing the electric charges.

Corona discharge is a powerful technique to modify the surface properties to create polymer electrets [5]. The slectric discharge of corona is obtained under atmospheric

Manuscript received on 6 July 2009, in final form 27 October 2009. pressure between two electrodes applied high dc voltage. The generated electric field excites gas molecules, and dissociates a part of them, which react with polymer molecules, induce the cleavage of chemical bonds and the formation of polar groups. Sellin et al. [6] treated the surface of PP with corona discharge, and investigated its chemical structure by Fourier transform infrared spectrophotometry (FTIR). They confirmed the presence of $\mathrm{COH}, \mathrm{C}=\mathrm{O}$, and $\mathrm{C}-\mathrm{O}$ groups on

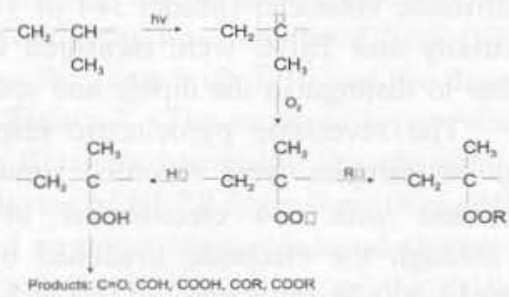

Figure 1. Reaction scheme of polypropylene by the poling process under the existence of oxygen proposed by Sellin et al. [6]. 
the surface, and proposed the reaction scheme during the corona discharge process as shown in Figure 1. It is now widely assumed that the surface oxidation process of PP induced by the corona discharge under air initiated by free radical reactions [7].

In recent researches, Lowkis et al [8] and Nifuku et al [9] observed strong homo charge polarization in PP foam electrets, and argued that PP treated with corona discharge owe its good piezo- and pyroelectric behavior to this homo charge. However, the contribution of hetero charge polarization induced by the orientation of polar groups at the surface of the PP films to the piezo- and pyroelectric behavior were also pointed out by Sellin et al [6]

In this study, the corona poling was carried out for the high and low crystalline PP films. The chemical structure and surface potential of the poled PP films were investigated by FTIR, thermally stimulated depolarization current (TSDC), pyroelectricity, differential scanning calorimetry (DSC), and gel permeation chromatography (GPC).

\section{EXPERIMENTAL}

\subsection{MATERIALS AND SURFACE TREATMENTS}

Two pellet samples of PP with different crystallinity were employed in this study. The sample with high crystallinity (HCPP) was BI750 manufactured by Samsung Atofina with the melting temperature of $165^{\circ} \mathrm{C}$, and the sample with low crystallinity (LCPP) was UBETAC manufactured by Ube Industries with the melting temperature of $100^{\circ} \mathrm{C}$. The pellets were pressed into films with the thickness of $10-30 \mu \mathrm{m}$ at $180^{\circ} \mathrm{C}$. To enhance the surface oxidation of samples, plasma treatment was performed to the PP films by dc discharge (air plasma) under $1.3 \mathrm{~Pa}\left(10^{-2}\right.$ Torr).

Poling was conducted by corona discharge in twoelectrode system of grounded plate electrode and corona electrode needle at $25-80^{\circ} \mathrm{C}$ under air or nitrogen gas. The voltage of the corona electrode was $\pm 5 \mathrm{kV}$, and the distance between the electrodes was $8 \mathrm{~mm}$. Electrical measurements except for the surface potential measurement were carried out for the samples metalized both sides by aluminum evaporation.

\subsection{MEASUREMENTS AND CHARACTERIZATION}

The surface potential of the poled samples was measured with an electrostatic voltmeter (Model 344 of Trek). Both the pyroelectricity and TSDC were measured for corona poled PP films to distinguish the dipole and space charge polarization. The reversible pyroelectric response and TSDC for poled samples were recorded simultaneously from the current with 614 electrometer of Keithley Instruments through the electrode irradiated by a pulse semiconductor laser $(670 \mathrm{~nm}, 3 \mathrm{~mW}, 10 \mathrm{~Hz})$ by heating at 4 ${ }^{\circ} \mathrm{C} \min ^{-1}$. The absolute value of the pyroelectric constant was determined from the reversible TSDC by heating and cooling near room temperature. The decay of the surface charge of poled samples was also monitored for 20 days.

While PP films without any electric treatments were insoluble to tetrahydrofuran (THF), the surface of the poled PP films can be dissolved into THF. The poled films were washed with THF to measure the weight loss by removing the oxidized layer of the films formed by the poling. The thickness of the oxidized layer was estimated from the weight loss and the density of PP $\left(0.95 \mathrm{~g} \mathrm{~cm}^{-3}\right)$.

The molecular weight calibrated with standard polystyrene samples of the components extracted with THF was determined with GPC-8020 system of Toso using THF as eluent.

In order to analyze the change of the chemical structure of PP films induced by corona treatment, FTIR was carried out with FTIR- 8900 of Shimadzu with a resolution of $4 \mathrm{~cm}^{-1}$ and 32 scans for a sample. The melting temperatures of the original sample and the component extracted with THF of HCPP were determined with DSC-60 system of Shimadzu. The samples were first cooled with liquid nitrogen, and heated from $-50^{\circ} \mathrm{C}$ to $160^{\circ} \mathrm{C}$ at the rate of $20^{\circ} \mathrm{C} / \mathrm{min}$.

\section{RESULTS}

\subsection{SURFACE POTENTIAL OF OXIDATION LAYER BY CORONA DISCHARGING}

Figure 2 shows the decay of the surface potential for HCPP films. The absolute values of the surface potential of films poled by plasma (positive), positive and negative corona reached almost constant values after 5 days. The surface potentials of the films poled by plasma, positive and negative corona were $750 \mathrm{~V}, 330 \mathrm{~V}$, and $-480 \mathrm{~V}$, respectively, 20 days after poling. Although the absolute values of the surface potential of the films poled both by positive and negative corona was so high, the former was a little smaller than the latter.

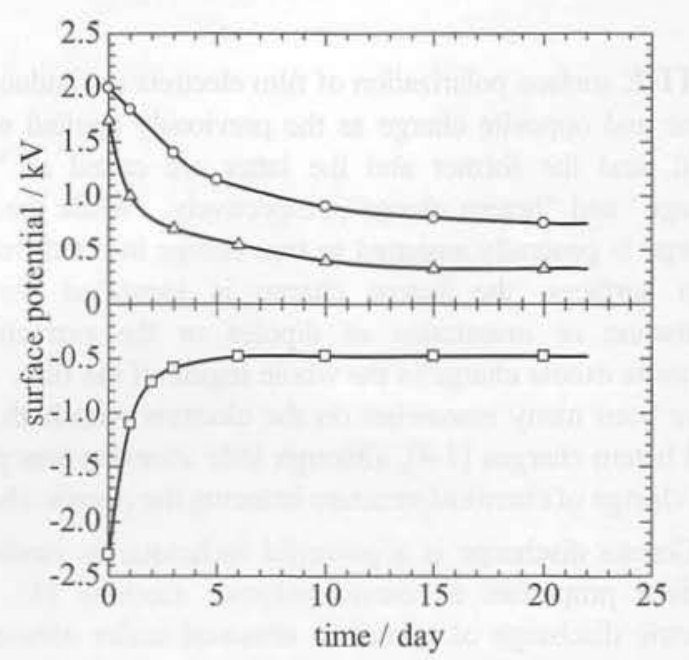

Figure 2. Time dependence of the surface potential of HCPP films treated by positive plasma (open circles), positive corona (open triangles), and negative corona (open squares) in the air. 
Figures 3-5 indicate the temperature dependence of TSDC and pyroelectricity of HCPP 20 days after the poling with different conditions. All the curves in Figures 3-5 had two peaks at $80-100{ }^{\circ} \mathrm{C}$ (hetero charge) and $120-140{ }^{\circ} \mathrm{C}$ (homo charge) with the opposite sign, which indicate that there were different relaxation modes for hetero and homo charges.

Figure 6 shows the temperature dependence of TSDC and pyroelectricity of LCPP 20 days after negative corona poling. The absolute values of TSDC and pyroelectricity of LCPP were 10 times smaller than those HCPP poled at the same condition (Figure 5), although the temperature dependence of TSDC and pyroelectricity of LCPP was essentially same as that of HCPP.

Table 1 summarizes the surface potential of PP films 20 days after poling at different conditions. Table 1 also indicates the lower surface potential of LCPP than that of HCPP. The reduction of the surface potential was observed when the poling process was conducted under nitrogen gas instead of the air. This shows that oxygen is required for the formation of

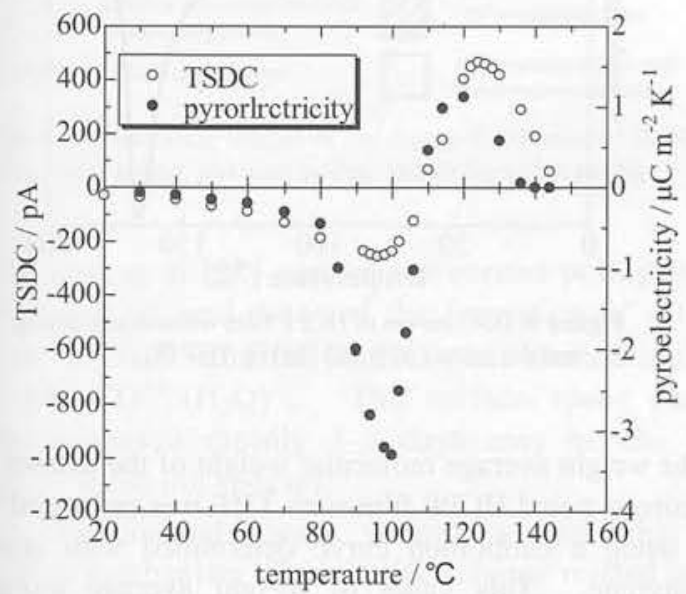

Figure 3. Temperature dependence of TSDC and pyroelectricity of HCPP 20 days after the poling by plasma in the air.



Figure 4. Temperature dependence of TSDC and pyroelectricity of HCPP 20 days after the treatment of positive corona poling in the air.

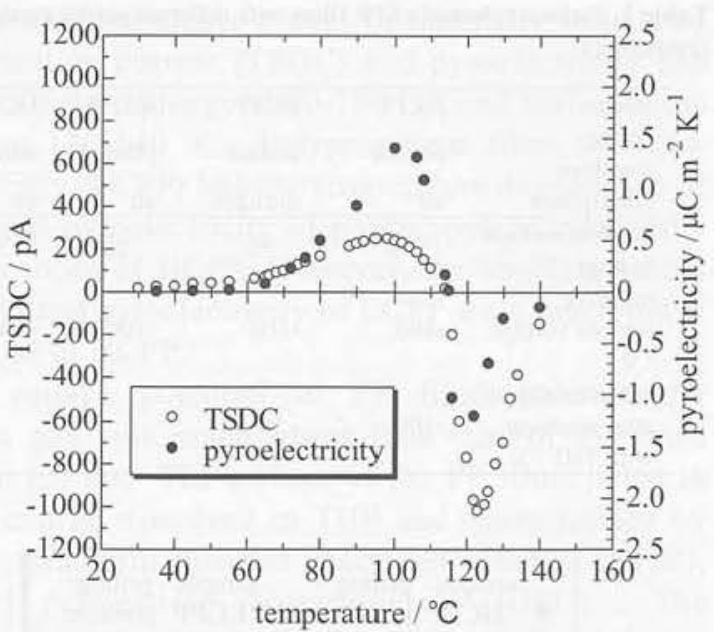

Figure 5. Temperature dependence of TSDC and pyroelectricity of HCPP 20 days after the treatment of negative corona poling in the air.

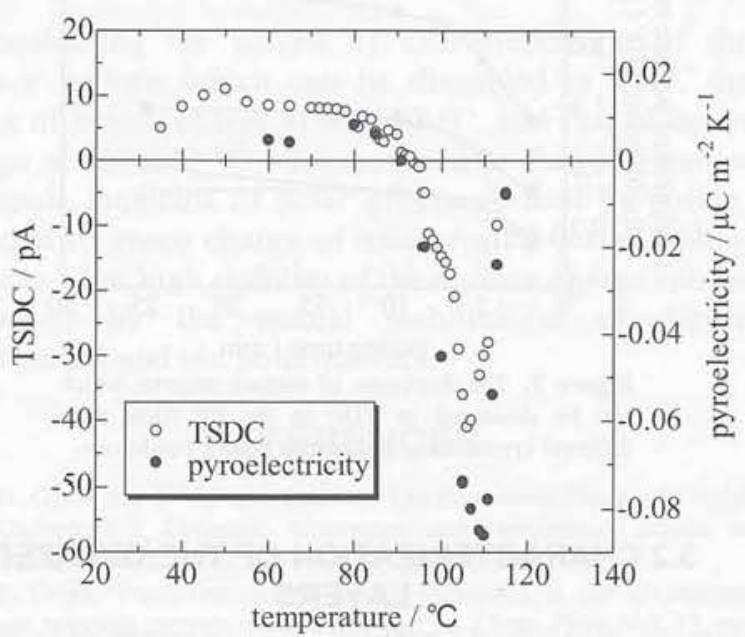

Figure 6. Temperature dependence of TSDC and pyroelectricity of LCPP 20 days after the treatment of negative corona poling in the air.

the surface layer as shown in Figure 1. The surface potentials of HCPP and LCPP films after washed with THF are also indicated in Table 1. Both the surface potential and pyroelectricity of LCPP were undetectable after washing with THF or mechanically scratching out the surface. The decrease of the surface potential was also detected for HCPP films, although the measured value scattered.

The thickness of the oxidized layer of PP films poled at different conditions was calculated from the weight loss by washing the films with THF and the density of PP as shown in Figure 7. The oxidized layers were thickened in all the films by increasing the charging time. The oxidized layers of HCPP films were thicker than those of LCPP, and negative charge induced thicker layers than positive charge. The tendency of the thickness of the oxidized layers well agreed with the absolute values of the surface potential shown in Figures 2-6 and Table 1. 
Table 1. Surface potential of PP films with different poling conditions and crystallinity.

\begin{tabular}{lllll}
\hline $\begin{array}{l}\text { sample } \\
\text { poling } \\
\text { condition } \\
\text { atmosphere }\end{array}$ & $\begin{array}{l}\text { HCPP } \\
\text { corona } \\
\text { air }\end{array}$ & $\begin{array}{l}\text { HCPP } \\
\text { corona } \\
\text { nitrogen }\end{array}$ & $\begin{array}{l}\text { HCPP } \\
\text { plasma } \\
\text { air }\end{array}$ & $\begin{array}{l}\text { LCPP } \\
\text { corona } \\
\text { air }\end{array}$ \\
\hline $\begin{array}{l}\text { positive surface } \\
\text { voltage /V } \\
\text { negative }\end{array}$ & 330 & 50 & 700 & 70 \\
$\begin{array}{l}\text { surface voltage } \\
\text { /V }\end{array}$ & -480 & -100 & -1000 & -100 \\
$\begin{array}{l}\text { surface voltage } \\
\text { after washing } \\
\text { with THF /V }\end{array}$ & -100 & -50 & & \\
\hline
\end{tabular}



Figure 7. The thickness of surface regions which can be dissolved in THF in the PP films with different crystallinity and corona poling conditions.

\subsection{CHARACTERIZATION OF THE OXIDIZED LAYERS}

Figure 8 shows the FTIR spectra of HCPP films of the untreated (a), 5 s corona treated (b), 30 s corona treated (c), and the extraction with THF (d). The absorption bands appeared at $3600-3200 \mathrm{~cm}^{-1}$ and $1720-1645 \mathrm{~cm}^{-1}$ in the spectrum (d) and small bands can be detected in the spectra (b) and (c). The former band was assigned as the stretching band of alcohol groups $(\mathrm{O}-\mathrm{H})$ forming hydrogen bonds, and the latter bands were caused by the stretching of carbonyl groups $(\mathrm{C}=\mathrm{O})$ of carboxyl groups $(\mathrm{COOH})$ and $\mathrm{C}=\mathrm{O}$ groups adjacent to olefinic double bonds or enolic $\mathrm{C}=\mathrm{O}$ groups in the treated films.

Figure 9 illustrates the DSC curves of HCPP without any electric treatment (a) and the extract from the corona poled HCPP film with THF (b). While the DSC curve of HCPP without any electric treatment had only single sharp endothermic peak at $165^{\circ} \mathrm{C}$, there were two broad peaks at 80 and $140{ }^{\circ} \mathrm{C}$ in the DSC curves of the extract from the corona poled HCPP film. As the peak temperatures in Figure 9 are roughly correspond with those of TSDC and pyroelectricity of poled PP films (Figures 3-6), the relaxations of TSDC and pyroelectricity of poled PP films may be dominated by the stability of the surface region of HCPP, which can be dissolved in THF.

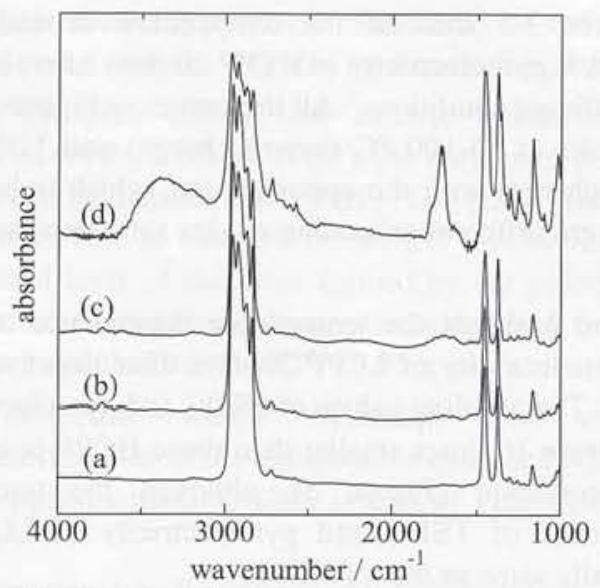

Figure 8. IR spectra of HCPP without any poling (a), a film treated $5 \mathrm{~s}$ of positive corona poling (b), a film treated $30 \mathrm{~s}$ of positive corona poling (c), and the extract of poled film by THF (d).

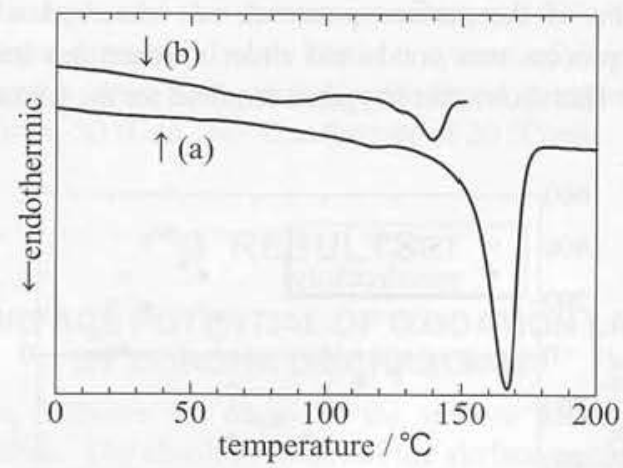

Figure 9. DSC curves of HCPP film without any poling (a), and the extract of poled film by THF (b).

The weight average molecular weight of the extract from the corona poled HCPP film with THF was estimated to be 246 using a calibration curve determined with standard polystyrene. This value of weight average molecular weight indicates that the HCPP in the surface layer created by the oxidization of poling was decomposed into oligomers with the degree of polymerization less than 10 .

\section{DISCUSSION}

The results described above indicate that electric treatments under the existence of oxygen formed oxidized layers at the surface of PP films. The PP molecules in the oxidized layers were decomposed into oligomers with polar groups such as $-\mathrm{OH}$ and $-\mathrm{C}=\mathrm{O}$, and can be dissolved in THF. The absolute values of surface charge were higher for the films with thicker oxidized layers. It should also be noted that the PP films with higher crystallinity had higher absolute values of surface charge and thicker oxidized layers. The temperature dependence of TSDC and pyroelectricity had the peak of hetero charge at $80-100{ }^{\circ} \mathrm{C}$, and that of homo charge at $120-140^{\circ} \mathrm{C}$, which indicates the existence of two different relaxation modes in TSDC and pyroelectricity. 
From the experimental results, we suppose the charge distribution as shown in the schematic image of Figure 10. The two peaks in the temperature dependence curves of TSDC and pyroelectricity in PP films indicate that the electric charges are trapped in two localized states [11]. As shown in Figure 2, the surface home charge decreased rapidly 1-5 days after poling, but remained constant values 5-20 days after poling. This time dependence requires the coexistence of electric charge diminished easily and that stabilized at least 20 days.

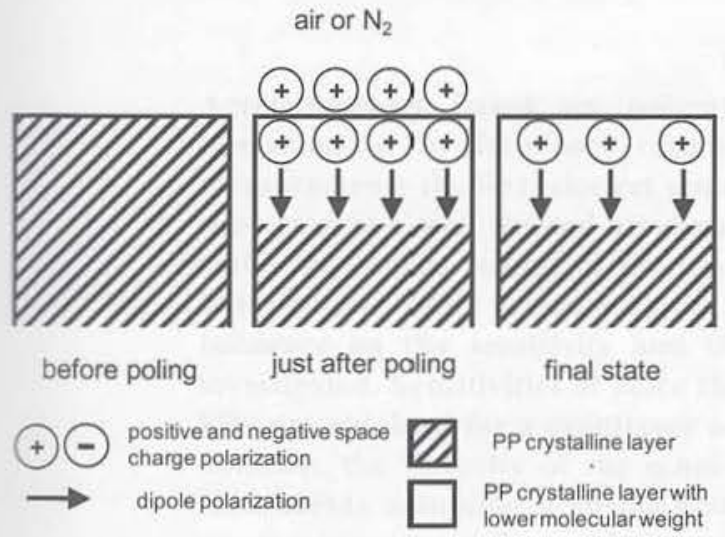

Figure 10. Schematic images of the charge distribution of HCPP films before poling, just after poling, and 20 days after poling.

Yovcheva et al [12] carried out corona poling to PP films in the air, and detected the formation of cations such as $\left(\mathrm{H}_{2} \mathrm{O}\right)_{n} \mathrm{H}^{+}$and $\left(\mathrm{H}_{2} \mathrm{O}\right)_{n} \mathrm{NO}^{+}$, and anions such as $\mathrm{CO}^{3-}$ and $\mathrm{CO}^{3-}\left(\mathrm{H}_{2} \mathrm{O}\right)_{n}$. The surface space charge which decreased rapidly 1-5 days may be the true charge of these ion species.

The experimental results strongly suggest that the stabilizing mechanism of the surface charge related to the surface oxidized layers which can be dissolved in THF. Whereas PP without any electric treatments is non-polar polymer, poled PP films had dipole moments caused by the polar groups detected by FTIR. Taking the result that the PP films with higher crystallinity had higher values of the absolute values of surface charge and thicker oxidized layers, it is inferred that the dipole moments of polar groups in the poled HCPP films (hetero charge) and the surface charge of ions (homo charge) stabilized each other, and had remained 20 days after poling. The low values of the surface potential in poled LCPP can be explained by that the dipole polarization is available in the regions with high crystallinity.

\section{CONCLUSION}

Both positive and negative corona poling was performed to polypropylene with high crystallinity (HCPP) films in the air. The surface potential of the films decreased 1-5 days after poling, but remained constant values after 5-20 days after poling. The temperature dependence curves of thermally stimulated depolarization current (TSDC) and pyroelectricity had a peak of hetero charge at $80-100{ }^{\circ} \mathrm{C}$, and that of homo charge at $120-140{ }^{\circ} \mathrm{C}$. Polypropylene films with low crystallinity (LCPP) had the temperature dependence of TSDC and pyroelectricity of polypropylene essentially same as those of HCPP. However, the absolute values of TSDC and pyroelectricity of LCPP were much lower than those of HCPP.

The surface potential of PP films poled under nitrogen gas was much lower than that of PP films poled in the air. The surface of the PP films poled in the air can be dissolved in THF and characterized by Fourier transform infrared spectrophotometry (FTIR), and gel permeation chromatography (GPC). The results of FTIR and GPC showed that the polymer chains at the surface of HCPP films were oxidized into oligomers with polar groups such as $\mathrm{COH}, \mathrm{C}=\mathrm{O}$, and C-O.

Considering the results of characterization of the surface regions which can be dissolved in THF, the peaks of hetero charge at $80-100{ }^{\circ} \mathrm{C}$, and that of homo charge at $120-140^{\circ} \mathrm{C}$ were assigned as the polarization of dipole moments of polar groups formed by poling, and that of space charge of ions created during poling process. The high stability of the surface charge can be explained by the mutual stabilization of dipole polarization and ion polarization.

\section{REFERENCES}

[1] B. Gross and L. Ferreira Denard, "On Permanent Charges in Solid Dielectrics I. Dielectric Absorption and Temperature Effects in Carnauba Wax", Phys. Rev. Vol. 67, pp. 253-259, 1945.

[2] B. Gross, "Permanent charges in solid dielectrics. II. Surface charges and transient currents in carnauba wax", J. Chem. Phys. Vol. 17, pp. 866-872, 1949.

[3] B. Gross and R. J. de Moraes, "Polarization of an electret", J. Chem. Phys. Vol. 37, pp. 710-713, 1962.

[4] G. M. Sessler and G. M. Yang, "Charge dynamics in electronirradiated polymers", Braz. J. Phys. Vol. 29, pp. 233-7240, 1999.

[5] V. Mangipudi, M.Tirrell, and A. V. Pocius, "Direct Measurement of the Surface Energy of Corona-Treated Polyethylene Using the Surface Forces Apparatus", Langmuir Vol. 11, pp. 19-23, 1995.

[6] N. Sellin, and J. S. Campos, "Surface composition analysis of PP films treated by corona discharge", "Mater. Res, Vol. 6, pp. 163-166, 2003.

[7] D. Briggs, C. R. Kendall, A. R. Blythe, and A. B. Wootton. "Electrical discharge treatment of polypropylene film", Polymer Vol. 24, pp. 47-52, 1983

[8] B. Lowkis, and E. Motyl, "Electret properties of polypropylene fabrics", J. Electrostatics, Vol. 51-52, pp. 232-238, 2001.

[9] M. Nifuku, Y. Zhou, A. Kisiel, T. Kobayashi, and H. Katoh, H., "Charging characteristics for electret filter materials", J. Electrostatics Vol. 51-52, pp. 200-205, 2001.

[10] X. Zhang, G. M. Sessler, and J. Hillenbrand, "Improvement of piezoelectric coefficient of cellular polypropylene films by repeated expansions", J. Electrostatics Vol. 65, pp. 94-100, 2007.

[11] M. Tang, Z. An, Z. Xia, and X. Zhang, "Electret properties and chemical modification of cellular polypropylene films", J. Electrostatics vol. 65, pp. 203-208, 2007.

[12] T. Yovcheva, G. Mekishev, and St. Nedev, "Corona electrets obtained in different gas media", J. Optoelectron. Adv. Mater. vol. 7, pp. $237-240,2005$. 


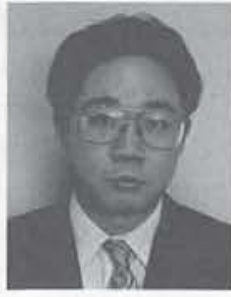

Yasuhiro Matsuda was born in Shizuoka, Japan in 1979, and graduated from the Department of Chemistry, Osaka University, Japan in 2002. He studied polymer physics and was awarded the Master (Science) degree in 2004 and the Doctor (Science) degree in 2006 at the same university. He was a postdoctoral fellow at the Institute for Materials Chemistry and Engineering, Kyushu University, Japan from 2006 to 2007 . He was appointed an assistant professor at the Department of Materials Science and Chemical Engineering, Shizuoka University in 2007. His present research interest is the relation between the solution properties and surface and interfacial properties of polymer.

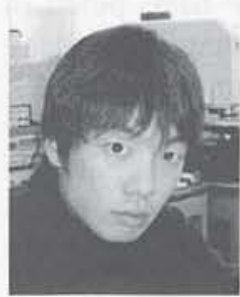

Yoshihiro Saito was born in Hamamatsu, Japan, in 1981. He received the B.E. degree in 2006, and the M.E. degree in 2008 from the Department of Materials Science and Chemical Engineering, Shizuoka University, Japan. He Joined Tokai Rubber Industries in 2008.

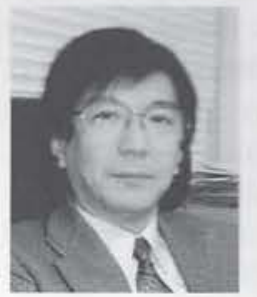

Shiperu Tasaka was born in Tokyo, Japan on 12 Octuber 1954. He received the B.E. degree in polymer engineering from Tokyo Univ, of A \& T in 1977 and the D.E. degree in polymer engineering from Tolyo Institute of Technology in 1984. He foined Shinsois University in 1987 and is now a professor in the Department of Materials Science and Chemical Engineering. His research activity is concemed with ferroelectric polymers and surface properties of polymers. $\mathrm{He}$ is a member of the Society of Polymer Science, Japan. 